\title{
Arbuscular Mycorrhizal (AM) fungal diversity on stabilized iron ore mine dumps in Goa, India
}

\author{
Tanvi N. Prabhu and B. F. Rodrigues* \\ Department of Botany, Goa University, Goa 403206. \\ *Corresponding author Email:felinov@gmail.com
}

(Submitted on November 30, 2019; Accepted on December 13,2019)

\begin{abstract}
The present study was carried out to explore arbuscular mycorrhizal (AM) fungal diversity on stabilized iron ore mine dumps in Goa. A total of 84 plant species belonging to 36 families were examined for the occurrence of AM fungal diversity in two study sites of which 21 plants were common to both sites. All the plants undertaken for study were found to be mycorrhizal. In our study $19 \mathrm{AM}$ fungal species belonging to eight genera, viz. Acaulospora, Funneliformis, Gigaspora, Glomus, Racocetra, Rhizophagus, Sclerocystis and Scutellospora were recovered. Acaulospora was dominant genus at both the sites. Based on Relative abundance (RA) and Isolation frequency (IF), Gigaspora albida was found to be dominant at site 1, while Scutellospora heterogama was dominant at site 2 .
\end{abstract}

KEYWORDS: restoration; spore density; root colonization; mine dumps.

\section{INTRODUCTION}

Land is one of the most essential natural resources on which human beings as well as all other terrestrial biomes depend. While society is being benefitted by the extraction of minerals it also causes significant environmental degradation. Mining disrupts the aesthetics of the landscape along with other soil components and results in the destruction of existing vegetation and soil profile (Kundu and Ghose, 1997). Thus, the mineral extraction process should ensure the return in productivity of the affected mines. The negative impacts of mining can be prevented by stabilizing a reject dump through revegetation (Ghose, 1989). Mined land sites are generally known to be nutrient deficient with low plant growth. Hence there is an urgent need to revegetate the mining sites so that there is establishment of stable nutrient cycles from plant growth and microbial process (Singh et al., 2002).

Arbuscular Mycorrhizal (AM) fungi are ubiquitous root symbionts colonizing $80 \%$ of the terrestrial plants (Wang and Qui, 2006). This symbiotic relationship benefits both the partners; the host plant provides the fungi with carbohydrates and in return receives mineral nutrients especially $\mathrm{P}$, increase in root surface area for absorption of water (Willis et al., 2013), plant field survival (Karthikeyan and Krishnakumar, 2012). They also improve soil structure, soil water relations, plant growth, yield and reduce fertilizer requirement (Finlay, 2008; Gianinazzi et al., 2010; Soka and Ritchie, 2014). Besides they are known to have a crucial role in plant community assembly and succession (Kikvidze et al., 2010).

These fungi as allied colonizers and biofertilizers could provide plants with benefits crucial for ecosystem restoration. It is important to use indigenous AM fungal strains which are best adapted to actual soil and climatic conditions to produce sitespecific AM fungus inocula. It has now become necessary to quantify the status of existence of indigenous AMF in degraded ecosystems (Mummey et al., 2002; Khan, 2004). Only few studies are being carried out to explore the diversity of AM fungi on iron ore mine wastelands of Goa (Rodrigues, 2000; Rodrigues and Bukhari, 1995). However, sites undertaken for the present investigation have not been studied previously. The objective of the present study was to assess the indigenous AM fungal diversity of ten year old mine sites in Goa, India.

\section{METHODOLOGY}

The study was carried out at two ten years old stabilized iron ore mines in Pale (North Goa) situated at geographic locations of $15^{\circ} 31^{\prime} 9.13^{\prime \prime} \mathrm{N}$ Latitude and $74^{\circ} 2^{\prime} 32.96^{\prime \prime} \mathrm{E}$ Longitude (Site 1) and $15^{\circ} 29^{\prime} 3.32^{\prime \prime N}$ Latitude and $74^{\circ}$ 3'35.55"E Longitude (Site 2). The plant species were identified with the help of local and regional floras (Vartak, 1966; Rao, 1985-86; Naithani et al., 1997). Root and rhizosphere soil samples were randomly collected from September 2014 to September 2016.

Three rhizosphere soil samples $(0-30 \mathrm{~cm})$ were collected for each plant species separately and brought to the laboratory in polyethylene bags. A composite soil sample was prepared by mixing the three soil samples of each plant species. After sieving sample for larger materials and root fragments, each sample was divided into six subsamples. Of these, three subsamples were used for spore extraction and the remaining three were used for soil analysis. Root fragments were used for estimation of AM colonization.

\section{Soil Analyses}

Soil $\mathrm{pH}$ was measured in soil water suspension $(40 \% \mathrm{w} / \mathrm{v})$ using $\mathrm{pH}$ meter (LI120 Elico, India). Electrical conductivity (EC) was measured by using a Conductivity meter (CM-180 Elico, India). Available nitrogen (N) was estimated using the method of Subbiah and Asija, (1956). Available phosphorus (P) was estimated using the method of Bray and Kurtz, (1945).The Hanway and Heidel method (1952) was used to estimate available Potassium (K) using Atomic Absorption Spectrophotometer (Nova 400P, Analytik Jena, Germany). Available micronutrients, viz. Zinc $(\mathrm{Zn})$, Copper $(\mathrm{Cu})$, Manganese (Mn) and Iron (Fe) were determined using DTPA $\mathrm{CaCl}_{2}$-TEA method of Lindsay and Norvell, (1978) using Atomic Absorption Spectrophotometer (AAS).

\section{Mycorrhizal colonization}

Root samples were hydrolyzed using $10 \% \mathrm{KOH}$ at $90^{\circ} \mathrm{C}$ for 2 hours followed by acidification in $5 \mathrm{~N} \mathrm{HCl}$ and staining with $0.05 \%$ trypan blue overnight (Phillips and Hayman, 1970). Later, stained roots were observed for colonization by mounting on a glass slide using Polyvinyl alcohol lacto glycerol (PVLG) as mountant. Slides were observed using bright field Olympus BX41 research microscope.The 
presence of hyphae, arbuscules and/or vesicles confirmed that the root segment was colonized. Per cent AM root colonization (RC) was calculated using the following formula (Readetal., 1976).

$$
\% \text { Root colonization }(\mathrm{RC})=\frac{\text { Number of root segments colonized }}{\text { Total number of root segments examined }} \times 100
$$

\section{Isolation and identification of $\mathrm{AM}$ fungal spores}

Spores were extracted from the rhizosphere soil subsamples separately for each plant using wet sieving and decanting method (Gerdemann and Nicolson, 1963). Extracted spores were mounted on glass slides in PVLG and were observed for spore morphology, wall characteristics, and dimensions under a bright field Olympus BX41 research microscope (40x, 100x and 400x).

The identification of AM fungi was carried out by using the relevant bibliographies (Rodrigues and Muthukumar 2009; Blaszkowski, 2012) and International Collection of Vesicular Arbuscular Mycorrhizal Fungi (INVAM). Names and epithets of AM fungal species were followed according to the recommendation of Schüßler and Walker (2010) and Redecker et al. (2013).

\section{Ecological and statistical data analysis}

The ecological characteristics of AM fungal species were estimated using the following indices:

a) Relative abundance $(\mathrm{RA})=$ (Number of spores of species/Total number of spores in all soil samples) $\mathrm{x}$ 100

b) Isolation frequency (IF) $=$ (Number of soil samples containing particular species/Total number of soil samples analysed) x 100

c) Shannon-Wiener diversity index $(\mathrm{H})=-\Sigma(P i \ln (P i))$, where $P i$ is the proportion of individual species that contributes to the total number of individuals(Shannon and Wiener, 1949).

d) Simpson`s diversity index (D) $=1-(\Sigma \mathrm{n}(\mathrm{n}-1) / \mathrm{N}(\mathrm{N}-1))$, where $n$ is the number of individuals of a given species and $\mathrm{N}$ is the total number of individuals in a community (Simpson, 1949).

e) Species richness $(\mathrm{SR})=$ total number of species in the community.

f) AMF species evenness (E): $\Sigma\left(H^{\prime}\right)=H^{\prime} / H^{\prime} \max$, where $H^{\prime} \max =\ln \mathrm{S}, \mathrm{S}=\mathrm{SR}$

Pearson coefficient of correlation $(r)$ was calculated to compare the relationship between spore density (SD) and RC, RA and IF and SD and SR using IBM SPSS Statistics 22 software.

\section{RESULTS}

Physico-chemical properties of lateritic soils of both the sites revealed that the soils were acidic in nature. All the plant macro- and micro-nutrients analysed were in low levels (Table1).
Table 1: Physico-chemical analyses of mine soils.

\begin{tabular}{|c|c|c|}
\hline \multirow{2}{*}{ Parameter } & \multicolumn{2}{|c|}{ Study sites } \\
\cline { 2 - 3 } & Site 1 & Site 2 \\
\hline $\mathrm{pH}$ & $5.45 \pm 0.04$ & $5.57 \pm 0.19$ \\
\hline $\mathrm{EC}(\mathrm{mS})$ & $0.01 \pm 0.12$ & $0.34 \pm 0.44$ \\
\hline $\mathrm{N}(\mathrm{g} / \mathrm{kg})$ & $0.08 \pm 0.04$ & $0.07 \pm 0.01$ \\
\hline $\mathrm{P}(\mathrm{g} / \mathrm{kg})$ & $0.01 \pm 0.00$ & $0.01 \pm 0.00$ \\
\hline $\mathrm{K}(\mathrm{g} / \mathrm{kg})$ & $0.02 \pm 0.00$ & $0.02 \pm 0.00$ \\
\hline $\mathrm{Zn}(\mathrm{ppm})$ & $3.25 \pm 0.47$ & $3.54 \pm 0.18$ \\
\hline $\mathrm{Cu}(\mathrm{ppm})$ & $0.03 \pm 0.02$ & $0.01 \pm 0.00$ \\
\hline $\mathrm{Fe}(\mathrm{ppm})$ & $21.73 \pm 2.67$ & $21.65 \pm 1.28$ \\
\hline $\mathrm{Mn}(\mathrm{ppm})$ & $101.77 \pm 2.05$ & $78.25 \pm 1.63$ \\
\hline $\mathrm{Ca}(\mathrm{ppm})$ & $623.15 \pm 6.86$ & $520.36 \pm 9.65$ \\
\hline
\end{tabular}

\section{Distribution of plant species diversity}

Atotal of 84 plants belonging to 36 families were surveyed from the selected sites. Of these, 21 plants were found to be common to both the sites (Table 2).

\section{AM colonization and spore density}

AM fungal colonization was recorded in roots of all sampled plant species from both the sites. Both arbuscular and vesicular colonization was recorded. Maximum RC from both the sites viz. site $1(86.67 \%)$ and site $2(80 \%)$ was recorded in Anacardium occidentale. The least RC was observed in Adiantum philippense (11.65\%) at site 1 and in Casuarina equisetifolia (25\%) at site 2 .

The highest SD was recorded in Lantana camara (304 spores/100g soil) at site 1 and in A. Occidentale (299 spores/100g soil) at site 2 . Lowest SD was observed in $A$. Philippense (8 spores/100g soil) at site 1 and in Pteris pellucida ( 8 spores $/ 100 \mathrm{~g}$ soil) at site 2 (Table 3 ).

\section{Diversity and Distribution of AM fungi}

A total of 19 AM fungal species belonging to eight genera were recorded from both the sites. Acaulospora (6) was found to be dominant genus followed by Gigaspora (4), Sclerocystis (3), Scutellospora (2), Funneliformis (1), Glomus (1), Racocetra (1), Rhizophagus (1) with species number given in parenthesis. Highest RA (29.72\%) and IF (56.46\%) were recorded for Gigaspora albida at site 1 and highest RA (35.09\%) and IF $(42.86 \%)$ for Scutellospora heterogama at site 2 .

Least RA $(0.15 \%)$ and $\operatorname{IF}(1.36 \%)$ was recorded in Sclerocystis rubiformis at site 1, while lowest RA $(0.13 \%)$ and IF $(1.19 \%)$ was recorded in Sclerocystis taiwanensis at site 2 (Table 4). Species richness (17) with species number given in parenthesis was recorded at both the sites.

Pearson's correlation coefficient showed that SD was significantly correlated with $\mathrm{RC}(\mathrm{r}=0.499, \mathrm{p}<0.01)$. However, no correlation was observed between SD and SR $(r=0.275)$. There existed a positive correlation between RA and IF $(r=$ $0.952, \mathrm{p}<0.01)$ at site 1 . A significant correlation existed between $\mathrm{SD}$ and $\mathrm{RC}(\mathrm{r}=0.310, \mathrm{p}<0.05)$, RA and IF $(\mathrm{r}=0.899$, $\mathrm{p}<0.01)$ and, SD and $\mathrm{SR}(\mathrm{r}=0.547, \mathrm{p}<0.01)$ on site 2 . ShannonWeiner index $(\mathrm{H})$, Simpson's index of dominance (D), and species evenness were higher on site 1 as compared to site 2 (Table 5). 
Table 2: Distribution of plant species recorded on a stabilized dump of both the iron ore mine sites.

\begin{tabular}{|c|c|c|}
\hline Family and Plant species & Habit & Location \\
\hline \multicolumn{3}{|l|}{ Acanthaceae } \\
\hline Andrographis paniculata (Burm.f.)Nees & Herb & 1 \\
\hline Lepidogathis lutea Dalz & Herb & 1 \\
\hline \multicolumn{3}{|l|}{ Amaranthaceae } \\
\hline Alternanthera sessilis (L.) R.Br. ex DC. & Herb & 1 \\
\hline Amaranthus viridis L. & Herb & 1 \\
\hline Celosia argentea $\mathrm{L}$. & Herb & 2 \\
\hline \multicolumn{3}{|l|}{ Anacardiaceae } \\
\hline Anacardium occidentale L. & Tree & 1,2 \\
\hline Mangifera indica L. & Tree & 1,2 \\
\hline \multicolumn{3}{|l|}{ Annonaceae } \\
\hline Annona squamosa $\mathrm{L}$. & Tree & 1,2 \\
\hline \multicolumn{3}{|l|}{ Apocynaceae } \\
\hline Allamanda cathartica L. & Shrub & 2 \\
\hline Alstonia scholaris (L.) R.Br. & Tree & 2 \\
\hline Hemidesmus indicus (L.) R.Br. & Twiner & 1 \\
\hline Rauvolfia serpentina (L.) Benth. ex Kurz & Shrub & 1 \\
\hline \multicolumn{3}{|l|}{ Asteraceae } \\
\hline Chromolaena odorata (L.) R.M.King \& H.Rob. & Herb & 1,2 \\
\hline Emilia sonchifolia (L.) DC. ex Wight & Herb & 1 \\
\hline Senecio bombayensis L. & Herb & 1 \\
\hline Tricholepis glaberrima DC & Herb & 1 \\
\hline Tridax procumbens $\mathrm{L}$. & Herb & 2 \\
\hline \multicolumn{3}{|l|}{ Balsaminaceae } \\
\hline Impatiens balsamina $\mathrm{L}$. & Herb & 1 \\
\hline Impatiens kleinii Wight \& Arn. & Herb & 1 \\
\hline \multicolumn{3}{|l|}{ Bixaceae } \\
\hline Bixa orellana L. & Shrub & 2 \\
\hline \multicolumn{3}{|l|}{ Cannabaceae } \\
\hline Trema orientalis (L.) Blume & Tree & 1,2 \\
\hline \multicolumn{3}{|l|}{ Casuarinaceae } \\
\hline Casuarina eqisetifolia $\mathrm{L}$. & Tree & 2 \\
\hline \multicolumn{3}{|l|}{ Clusiaceae } \\
\hline Garcinia indica Choiss. & Tree & 2 \\
\hline \multicolumn{3}{|l|}{ Colchicaceae } \\
\hline Gloriosa superba L. & Climber & 1 \\
\hline \multicolumn{3}{|l|}{ Combretaceae } \\
\hline Calycopteris floribunda (Roxb.) Lam.ex Poir & Shrub & 1,2 \\
\hline \multicolumn{3}{|l|}{ Commelinaceae } \\
\hline Commelina diffusa Burm.f. & Herb & 2 \\
\hline Murdannia semiteres(Dalzell) Santapau & Herb & 1,2 \\
\hline \multicolumn{3}{|l|}{ Cyperaceae } \\
\hline Cyperus iria $\mathrm{L}$. & Herb & 2 \\
\hline Cyperus rotandus $\mathrm{L}$. & Herb & 2 \\
\hline \multicolumn{3}{|l|}{ Euphorbiaceae } \\
\hline Macaranga peltata Roxb. Mueller & Tree & 1,2 \\
\hline Ricinus communis L. & Shrub & 2 \\
\hline \multicolumn{3}{|l|}{ Fabaceae } \\
\hline Acacia auriculiformis A.Cunn. ex Benth. & Tree & 1,2 \\
\hline Acacia mangium Willd. & Tree & 1,2 \\
\hline Alysicarpus vaginalis (L.) DC. & Herb & 1 \\
\hline Cassia fistula $\mathrm{L}$. & Tree & 1,2 \\
\hline Senna siamea (Lam.) Irwin et Barneby & Tree & 2 \\
\hline Senna tora (L.) Roxb. & Herb & 1,2 \\
\hline Crotalaria filipesBenth. & Herb & 1 \\
\hline Crotalaria pallida $\mathrm{L}$. & Herb & 1 \\
\hline Delonix regia (Boj. ex Hook.) Raf. & Tree & 2 \\
\hline Gliricidia sepium (Jacq.) Kunth ex Walp. & Tree & 2 \\
\hline Leucaena leucocephala (Lam.) de Wit & Tree & 1,2 \\
\hline Mimosa pudica L. & Herb & 1,2 \\
\hline
\end{tabular}

Legends: $1=$ Site $1 ; 2=$ Site 2

\section{DISCUSSION}

The study revealed that the soil of both the mine dumps was acidic in nature with very low levels of plant macro and micro-nutrients. Similar observations were recorded earlier by Rodrigues (2000). Nutrient deficiency is a primary

\begin{tabular}{|c|c|c|}
\hline Family and Plant species & Habit & Location \\
\hline \multicolumn{3}{|l|}{ Fabaceae } \\
\hline Peltophorum pterocarpum (DC.) K.Heyne & Tree & 2 \\
\hline Pithocellobium dulce(Roxb.) Benth. & Tree & 2 \\
\hline Pongamia pinnata (L.) Panigrahi & Tree & 1 \\
\hline Smithia confertaSm. & Herb & 1,2 \\
\hline Stylosanthes hamata (L.) Taub. & Herb & 1,2 \\
\hline Tamarindus indica $\mathrm{L}$. & Tree & 2 \\
\hline \multicolumn{3}{|l|}{ Gentianaceae } \\
\hline \multicolumn{3}{|l|}{ Lamiaceae } \\
\hline Gmelina arborea Roxb. & Tree & 2 \\
\hline Leucas aspera(Willd.) Link & Herb & 2 \\
\hline \multicolumn{3}{|l|}{ Leguminosae } \\
\hline Phanera purpurea (L.) Benth. & Tree & 2 \\
\hline \multicolumn{3}{|l|}{ Linderniaceae } \\
\hline \multicolumn{3}{|l|}{ Lythraceae } \\
\hline \multicolumn{3}{|l|}{ Malvaceae } \\
\hline Bombax ceiba $\mathrm{L}$. & Tree & 2 \\
\hline $\begin{array}{l}\text { Gossypium arboreum } \mathrm{L} . \\
\text { Meliaceae }\end{array}$ & \multicolumn{2}{|c|}{ Meliaceae } \\
\hline \multirow{2}{*}{\multicolumn{3}{|c|}{ Moraceae }} \\
\hline & & \\
\hline Artocarpus altilis (Parkinson) Fosberg & Tree & 2 \\
\hline Artocarpus heterophyllus Lam. & Tree & 1,2 \\
\hline Ficus hispida L.f. & Tree & 1 \\
\hline \multicolumn{3}{|l|}{ Myrtaceae } \\
\hline Psidium guajava $\mathrm{L}$. & Tree & 2 \\
\hline Syzygium cumini (L.) Skeels & Tree & 2 \\
\hline \multicolumn{3}{|l|}{ Orobanchaceae } \\
\hline $\begin{array}{l}\text { Rhamphicarpa fistulosa (Hochst.) Benth. } \\
\text { Phyllanthaceae }\end{array}$ & Herb & 1 \\
\hline Phyllanthus emblica $\mathrm{L}$. & Tree & 2 \\
\hline \multicolumn{3}{|l|}{ Plantaginaceae } \\
\hline Scoparia dulcis $\mathrm{L}$. & Herb & 2 \\
\hline \multicolumn{3}{|l|}{ Poaceae } \\
\hline Cymbopogon citratus (DC.) Stapf & Herb & 2 \\
\hline Cynodon dactylon (L.) Pers. & Herb & 2 \\
\hline Dactyloctenium aegyptium (L.) Willd. & Herb & 1 \\
\hline Digitaria ciliaris (Retz.) Koeler & Herb & 1 \\
\hline Eleusine indica (L.) Gaertn. & Herb & 1 \\
\hline Eragrostis uniloides (Retzius) Nees ex Steudel & Herb & 1 \\
\hline Panicum notatum Hack & Herb & 1 \\
\hline Panicumsp. & Herb & 2 \\
\hline $\begin{array}{l}\text { Pennisetum hohenackeri Hochst. ex Steud. } \\
\text { Pteridaceae }\end{array}$ & Herb & 2 \\
\hline Adiantum philippense L. & Herb & 1 \\
\hline Cheilanthes micropteria $\mathrm{Sw}$. & Herb & 1 \\
\hline Pteris pellucida $\mathrm{L}$. & Herb & 2 \\
\hline \multicolumn{3}{|l|}{ Rhamnaceae } \\
\hline \multirow{2}{*}{\multicolumn{3}{|c|}{ Rutaceae }} \\
\hline & & \\
\hline Citrus limon (L.) Osbeck & Tree & 1 \\
\hline \multicolumn{2}{|l|}{ Sapotaceae } & 1,2 \\
\hline $\begin{array}{l}\text { Mimusops elengi } \mathrm{L} \text {. } \\
\text { Simaroubaceae }\end{array}$ & Tree & 2 \\
\hline Simarouba glauca DC. & Tree & 2 \\
\hline Verbenaceae & & \\
\hline Lantana camara $\mathrm{L}$. & Herb & 1,2 \\
\hline
\end{tabular}

limiting factor for plant growth on mining impacted sites. Overburdened rejected dumps are known to be deficient in plant macronutrients (Sheoran et al.,2008;2010).

In this study a total of 19 AM fungal species belonging to 8 genera were recovered from the rhizosphere soils of 84 plant 
Table 3: Per cent root colonization and spore density of AM fungi on the selected mine sites.

\begin{tabular}{|c|c|c|c|c|}
\hline \multirow[t]{2}{*}{ Family and Plant species } & \multicolumn{2}{|c|}{ Colonization \% } & \multicolumn{2}{|c|}{ Spore density \% } \\
\hline & Site 1 & Site 2 & Site 1 & Site 2 \\
\hline \multicolumn{5}{|l|}{ Acanthaceae } \\
\hline Andrographis paniculata (Burm.f.)Nees & $53.33 \pm 5.81$ & - & $16.67 \pm 1.76$ & - \\
\hline Lepidogathis lutea Dalz. & $42.67 \pm 3.71$ & - & $22.67 \pm 5.21$ & - \\
\hline \multicolumn{5}{|l|}{ Amaranthaceae } \\
\hline Alternanthera sessilis (L.) R.Br. ex DC. & $55.00 \pm 10.41$ & - & $30.33 \pm 2.91$ & - \\
\hline Amaranthus viridis L. & $45.00 \pm 7.64$ & - & $31.67 \pm 2.73$ & - \\
\hline Celosia argentea L. & - & $64.00 \pm 2.52$ & - & $19.67 \pm 1.45$ \\
\hline \multicolumn{5}{|l|}{ Anacardiaceae } \\
\hline Anacardium occidentale L. & $86.67 \pm 6.96$ & $80.00 \pm 1.15$ & $168.33 \pm 3.53$ & $299.33 \pm 5.81$ \\
\hline Mangifera indica L. & $85.00 \pm 2.89$ & $69.33 \pm 3.48$ & $49.67 \pm 8.97$ & $20.33 \pm 4.18$ \\
\hline \multicolumn{5}{|l|}{ Annonaceae } \\
\hline Annona squamosa L. & $36.67 \pm 3.33$ & $64.33 \pm 3.48$ & $41.33 \pm 7.75$ & $81.33 \pm 9.24$ \\
\hline \multicolumn{5}{|l|}{ Apocynaceae } \\
\hline Allamanda cathartica L. & - & $45.33 \pm 3.53$ & - & $22.00 \pm 3.06$ \\
\hline Alstonia scholaris (L.) R.Br. & - & $56.67 \pm 4.63$ & - & $108.67 \pm 3.48$ \\
\hline Hemidesmus indicus (L.) R.Br. & $56.67 \pm 4.67$ & - & $25.00 \pm 3.00$ & - \\
\hline Rauvolfia serpentina (L.) Benth. ex Kurz. & $35.00 \pm 5.00$ & -- & $21.00 \pm 1.15$ & \\
\hline \multicolumn{5}{|l|}{ Asteraceae } \\
\hline Chromolaena odorata (L.) R.M.King \& H.Rob. & $76.67 \pm 4.41$ & $29.33 \pm 2.91$ & $14.33 \pm 4.48$ & $45.67 \pm 1.45$ \\
\hline Emilia sonchifolia (L.) DC. ex Wight & $78.00 \pm 3.46$ & - & $24.33 \pm 2.85$ & - \\
\hline Senecio bombayensis L. & $52.00 \pm 4.62$ & - & $10.33 \pm 1.33$ & - \\
\hline Tricholepis glaberrima DC. & $45.33 \pm 4.67$ & - & $11.00 \pm 2.08$ & - \\
\hline Tridax procumbens L. & & $66.67 \pm 4.41$ & - & $31.33 \pm 4.06$ \\
\hline \multicolumn{5}{|l|}{ Balsaminaceae } \\
\hline Impatiens balsamina $\mathrm{L}$. & $68.00 \pm 4.62$ & - & $25.67 \pm 2.60$ & - \\
\hline Impatiens kleinii Wight \& Arn. & $42.00 \pm 7.02$ & & $25.33 \pm 3.71$ & - \\
\hline \multicolumn{5}{|l|}{ Bixaceae } \\
\hline Bixa orellana L. & - & $35.00 \pm 2.89$ & - & $62.33 \pm 2.60$ \\
\hline \multicolumn{5}{|l|}{ Cannabaceae } \\
\hline Trema orientalis (L.) Blume & $80.67 \pm 4.06$ & $68.00 \pm 6.11$ & $79.33 \pm 4.67$ & $90.00 \pm 4.62$ \\
\hline \multicolumn{5}{|l|}{ Casuarinaceae } \\
\hline Casuarina equisetifolia $\mathrm{L}$. & - & $25.00 \pm 5.77$ & - & $23.67 \pm 4.33$ \\
\hline \multicolumn{5}{|l|}{ Clusiaceae } \\
\hline Garcinia indica Choiss. & - & $49.33 \pm 1.76$ & - & $105.67 \pm 5.21$ \\
\hline \multicolumn{5}{|l|}{ Colchicaceae } \\
\hline Gloriosa superba L. & $18.33 \pm 4.41$ & - & $26.33 \pm 3.84$ & - \\
\hline \multicolumn{5}{|l|}{ Combretaceae } \\
\hline Calycopteris floribunda (Roxb.) Lam.ex Poir & $16.65 \pm 6.01$ & $53.33 \pm 12.02$ & $26.33 \pm 3.84$ & $59.00 \pm 3.46$ \\
\hline Commelinaceae & & & & \\
\hline Commelina diffusa Burm.f. & - & $33.33 \pm 6.01$ & - & $22.67 \pm 2.40$ \\
\hline Murdannia semiteres (Dalzell) Santapau & $23.33 \pm 8.33$ & $34.67 \pm 2.40$ & $16.00 \pm 2.52$ & $24.33 \pm 4.48$ \\
\hline Cyperaceae & & & & \\
\hline Cyperus iriaL. & - & $63.33 \pm 7.26$ & - & $31.00 \pm 1.53$ \\
\hline Cyperus rotandus $\mathrm{L}$. & -- & $43.33 \pm 8.82$ & & $25.00 \pm 3.51$ \\
\hline Euphorbiaceae & & & & \\
\hline Macaranga peltata Roxb. Mueller & $55.33 \pm 3.53$ & $51.00 \pm 4.93$ & $68.33 \pm 4.91$ & $16.67 \pm 4.91$ \\
\hline Ricinus communis L. & - & $62.67 \pm 1.45$ & - & $63.00 \pm 8.62$ \\
\hline Fabaceae & & & & \\
\hline Acacia auriculiformis A.Cunn. ex Benth. & $31.67 \pm 6.07$ & $30.00 \pm 4.16$ & $44.00 \pm 2.65$ & $22.67 \pm 2.67$ \\
\hline Acacia mangium Willd. & $33.33 \pm 4.41$ & $26.67 \pm 9.28$ & $18.33 \pm 3.71$ & $48.33 \pm 8.35$ \\
\hline Alysicarpus vaginalis (L.) DC. & $36.33 \pm 3.28$ & - & $19.67 \pm 3.28$ & - \\
\hline Casia fistula $\mathrm{L}$. & $72.00 \pm 3.06$ & $78.33 \pm 4.41$ & $54.33 \pm 4.10$ & $76.67 \pm 4.98$ \\
\hline Senna siamea (Lam.) Irwin et Barneby & - & $31.00 \pm 4.16$ & - & $41.67 \pm 2.40$ \\
\hline Senna tora (L.) Roxb. & $66.67 \pm 2.91$ & $51.00 \pm 3.61$ & $96.33 \pm 3.84$ & $80.33 \pm 3.93$ \\
\hline Crotalaria filipes Benth. & $35.00 \pm 2.89$ & - & $17.00 \pm 3.21$ & - \\
\hline Crotalaria pallida $\mathrm{L}$. & $35.33 \pm 6.36$ & -- & $13.00 \pm 1.53$ & \\
\hline Delonix regia (Boj. ex Hook.) Raf. & - & $58.33 \pm 13.02$ & - & $46.00 \pm 11.59$ \\
\hline Gliricidia sepium (Jacq.) Kunth ex Walp. & - & $40.00 \pm 8.66$ & - & $65.67 \pm 11.57$ \\
\hline Leucaena leucocephala (Lam.) de Wit & $31.67 \pm 8.82$ & $65.00 \pm 7.64$ & $25.33 \pm 4.48$ & $34.33 \pm 6.77$ \\
\hline Mimosa pudica $\mathrm{L}$. & $46.67 \pm 7.26$ & $28.67 \pm 2.03$ & $34.67 \pm 8.74$ & $24.00 \pm 4.00$ \\
\hline
\end{tabular}




\begin{tabular}{|c|c|c|c|c|}
\hline \multirow[t]{2}{*}{ Family and Plant species } & \multicolumn{2}{|c|}{ Colonization \% } & \multicolumn{2}{|c|}{ Spore density \% } \\
\hline & Site 1 & Site 2 & Site 1 & Site 2 \\
\hline \multicolumn{5}{|l|}{ Fabaceae } \\
\hline Peltophorum pterocarpum (DC.) K.Heyne & - & $55.00 \pm 2.89$ & - & $41.00 \pm 8.74$ \\
\hline Pithocellobium dulce (Roxb.) Benth. & - & $54.67 \pm 2.40$ & - & $65.33 \pm 4.06$ \\
\hline Pongamia pinnata (L.) Panigrahi & $31.67 \pm 1.76$ & - & $57.67 \pm 3.84$ & - \\
\hline Smithia conferta $\mathrm{Sm}$. & $60.00 \pm 10.41$ & $36.67 \pm 9.28$ & $15.00 \pm 3.46$ & $59.33 \pm 3.18$ \\
\hline Stylosanthes hamata (L.) Taub. & $46.00 \pm 4.00$ & $39.67 \pm 0.88$ & $35.00 \pm 4.51$ & $36.67 \pm 5.70$ \\
\hline Tamrindus indica $\mathrm{L}$. & - & $41.33 \pm 4.37$ & - & $78.33 \pm 2.91$ \\
\hline \multicolumn{5}{|l|}{ Gentianaceae } \\
\hline $\begin{array}{l}\text { Canscora diffusa (Vahl) R.Br. ex Roem. \& } \\
\text { Schult. }\end{array}$ & $71.33 \pm 3.53$ & $60.00 \pm 7.64$ & $50.33 \pm 3.76$ & $34.00 \pm 5.29$ \\
\hline \multicolumn{5}{|l|}{ Lamiaceae } \\
\hline Gmelina arborea Roxb. & - & $61.67 \pm 4.41$ & - & $52.00 \pm 5.13$ \\
\hline Leucas aspera (Willd.) Link. & - & $47.00 \pm 4.36$ & - & $16.67 \pm 4.91$ \\
\hline \multicolumn{5}{|l|}{ Leguminosae } \\
\hline Phanera purpurea (L.) Benth. & - & $39.67 \pm 2.03$ & - & $33.67 \pm 3.18$ \\
\hline \multicolumn{5}{|l|}{ Linderniaceae } \\
\hline Lindernia crustacea (L.) F. Muell. & $43.33 \pm 13.02$ & $30.00 \pm 7.64$ & $10.00 \pm 1.53$ & $26.00 \pm 6.43$ \\
\hline \multicolumn{5}{|l|}{ Lythraceae } \\
\hline Punica granatum L. & $18.33 \pm 1.67$ & - & $12.33 \pm 2.85$ & - \\
\hline \multicolumn{5}{|l|}{ Malvaceae } \\
\hline Bombax ceiba L. & - & $61.33 \pm 1.86$ & - & $36.67 \pm 2.60$ \\
\hline Gossypium arboreum L. & - & $68.33 \pm 10.93$ & - & $25.67 \pm 3.38$ \\
\hline \multicolumn{5}{|l|}{ Meliaceae } \\
\hline Azadirachta indica A.Juss. & - & $58.33 \pm 3.18$ & - & $85.67 \pm 3.38$ \\
\hline \multicolumn{5}{|l|}{ Moraceae } \\
\hline Artocarpus alitis (Parkinson) Fosberg & - & $63.33 \pm 9.28$ & - & $33.00 \pm 11.59$ \\
\hline Artocarpus heterophyllus Lam. & $63.33 \pm 7.26$ & $42.33 \pm 2.85$ & $69.33 \pm 5.36$ & $15.67 \pm 4.91$ \\
\hline Ficus hispida L.f. & $53.33 \pm 6.01$ & - & $41.00 \pm 8.08$ & - \\
\hline \multicolumn{5}{|l|}{ Myrtaceae } \\
\hline Psidium guajava $\mathrm{L}$. & - & $48.67 \pm 1.76$ & - & $24.00 \pm 4.62$ \\
\hline Sygzgium cumini (L.) Skeels & - & $38.00 \pm 4.73$ & - & $50.33 \pm 6.69$ \\
\hline \multicolumn{5}{|l|}{ Orobanchaceae } \\
\hline Rhamphicarpa fistulosa (Hochst.) Benth. & $23.33 \pm 4.41$ & - & $14.67 \pm 3.28$ & - \\
\hline \multicolumn{5}{|l|}{ Phyllanthaceae } \\
\hline Phyllanthus emblica L. & - & $43.67 \pm 1.86$ & - & $40.67 \pm 4.91$ \\
\hline Plantaginaceae & & & & \\
\hline Scoparia dulcis L. & - & $38.33 \pm 9.28$ & - & $21.33 \pm 2.91$ \\
\hline Poaceae & & & & \\
\hline Cymbopogon citratus (DC.) Stapf & - & $73.33 \pm 4.41$ & - & $52.00 \pm 8.89$ \\
\hline Cynodon dactylon (L.) Pers. & - & $79.33 \pm 0.67$ & - & $20.67 \pm 3.28$ \\
\hline Dactyloctenium aegyptium (L.) Willd. & $56.67 \pm 9.28$ & - & $31.67 \pm 3.67$ & - \\
\hline Digitaria ciliaris (Retz.) Koeler & $60.00 \pm 10.41$ & - & $30.00 \pm 1.53$ & - \\
\hline Eleusine indica (L.) Gaertn. & $31.67 \pm 6.01$ & - & $13.00 \pm 4.36$ & - \\
\hline Eragrostis uniloides (Retzius) Nees ex Steudel & $35.00 \pm 5.00$ & - & $21.00 \pm 3.61$ & - \\
\hline Panicum notatum Hack & $48.33 \pm 10.14$ & - & $12.33 \pm 2.85$ & - \\
\hline Panicum sp. & - & $46.67 \pm 3.53$ & - & $41.00 \pm 4.36$ \\
\hline Pennisetum hohenackeri Hochst. ex Steud. & - & $71.67 \pm 7.26$ & - & $31.33 \pm 7.06$ \\
\hline Pteridaceae & & & & \\
\hline Adiantum philippense L. & $11.65 \pm 1.67$ & - & $8.33 \pm 0.88$ & - \\
\hline Cheilanthes micropteria Sw. & $26.67 \pm 2.91$ & - & $20.00 \pm 1.15$ & - \\
\hline Pteris pellucida $\mathrm{L}$. & - & $60.00 \pm 7.64$ & - & $8.67 \pm 1.20$ \\
\hline Rhamnaceae & & & & \\
\hline Zizyphus mauritiana Lam. & $63.33 \pm 8.82$ & - & $19.33 \pm 6.44$ & - \\
\hline Rutaceae & & & & \\
\hline Citrus limon (L.) Osbeck & $23.35 \pm 7.26$ & - & $14.66 \pm 3.28$ & - \\
\hline Sapotaceae & & & & \\
\hline Manilkara zapota (L.) P.Royen & $28.30 \pm 6.01$ & $46.67 \pm 2.33$ & $26.00 \pm 6.56$ & $36.67 \pm 2.03$ \\
\hline Mimusops elengi L. & - & $38.00 \pm 3.06$ & - & $55.67 \pm 3.76$ \\
\hline Simaroubaceae & & & & \\
\hline Simarouba glauca DC. & - & $58.00 \pm 5.69$ & - & $96.67 \pm 6.89$ \\
\hline Verbenaceae & & & & \\
\hline Lantana camara $\mathrm{L}$. & $76.00 \pm 5.29$ & $65.33 \pm 3.71$ & $304.00 \pm 3.21$ & $72.00 \pm 6.08$ \\
\hline
\end{tabular}


Table 4: Relative Abundance (RA) and Isolation frequency (IF) of AM fungi on the selected mine sites.

\begin{tabular}{|c|c|c|c|c|}
\hline \multirow[t]{2}{*}{ AM fungal species } & \multicolumn{2}{|c|}{ RA \% } & \multicolumn{2}{|c|}{ IF\% } \\
\hline & Site 1 & Site 2 & Site 1 & Site 2 \\
\hline Acaulospora delicata Walker, Pfeiff. \& Bloss & 4.53 & 5.01 & 15.65 & 17.86 \\
\hline Acaulospora dilatata Morton & 1.82 & 1.21 & 10.20 & 5.95 \\
\hline $\begin{array}{l}\text { Acaulospora myriocarpa Spain, Sieverd. \& } \\
\text { Schenck, }\end{array}$ & 1.16 & 3.03 & 4.08 & 8.33 \\
\hline Acaulospora rehmii Sieverd. \& Toro & 4.97 & 7.03 & 16.33 & 20.24 \\
\hline Acaulospora scrobiculata Trappe & 7.26 & 6.85 & 12.93 & 29.76 \\
\hline Acaulospora undulata Sieverd. & 5.28 & 2.39 & 13.61 & 8.33 \\
\hline $\begin{array}{l}\text { Funneliformis geosporum (Nicolson \& Gerd.) } \\
\text { Walker \& Schüßler }\end{array}$ & 0.38 & 0.59 & 2.04 & 2.98 \\
\hline Gigaspora albida Schenck \& Sm. & 29.72 & 21.99 & 56.46 & 41.07 \\
\hline Gigaspora decipiens Hall \& Abbott & 10.43 & 2.06 & 19.73 & 3.57 \\
\hline $\begin{array}{l}\text { Gigaspora gigantea (Nicolson \& Gerd.) Gerd. } \\
\text { \& Trappe }\end{array}$ & - & 3.52 & - & 7.14 \\
\hline Gigaspora margarita Becker \& Hall & 3.48 & 1.80 & 8.16 & 5.36 \\
\hline Glomus macrocarpum Tul. \& Tul. & 0.36 & 0.63 & 2.04 & 4.17 \\
\hline $\begin{array}{l}\text { Racocetra gregaria } \text { (Schenck \& Nicolson) } \\
\text { Oeh1, Souza \& Sieverd. }\end{array}$ & 3.08 & 6.68 & 10.88 & 8.93 \\
\hline $\begin{array}{l}\text { Rhizophagus fasciculatus (Thaxt.) Gerd. \& } \\
\text { Trappe }\end{array}$ & 0.96 & - & 3.40 & - \\
\hline Sclerocystis rubiformis Gerd. \& Trappe & 0.15 & 0.13 & 1.36 & 2.38 \\
\hline Sclerocystis sinuosa Gerd. \& Bakshi & 0.25 & - & 2.72 & - \\
\hline Sclerocystis taiwanensis Wu \& Chen & - & 0.06 & - & 1.19 \\
\hline $\begin{array}{l}\text { Scutellospora calospora(Nicolson \& Gerd.) } \\
\text { Walker \& Sanders }\end{array}$ & 4.02 & 1.94 & 10.88 & 3.57 \\
\hline $\begin{array}{l}\text { Scutellospora heterogama (Nicolson \&Gerd.) } \\
\text { Walker \& Sanders }\end{array}$ & 21.44 & 35.09 & 25.85 & 42.86 \\
\hline
\end{tabular}

species belonging to 36 families. Mycorrhizal symbiosis plays a crucial role in survival and nutrient uptake of plants especially in P deficient derelict soils (Khan, 2005). However, very low $P$ availability is responsible to inhibit AM colonization (Tinker, 1975; Bolan, 1991; de Miranda and Harris, 1994).

Acaulospora was the dominant genus on both the study sites. The acidic nature of the reject dumps may explain the dominance of the genus Acaulospora. According to Giovannetti et al., (2010) the genus Acaulospora is predominant in acidic soils. However, this is contradictory to the results in earlier studies (Jasper et al., 1988; Sastry and Johri, 1999; Sharma et al., 2009; Kullu and Bahera, 2012) who recorded Glomus to be the dominant genus on the mine spoil dump. However, our study revealed that in terms of RA and IF, Gi. albida and Sc. heterogama were dominant species on site 1 and site 2, respectively. This dominance may be due to change in host's nutritional demands in the developmental stages as AM species that colonise the host in early stages become minor and are replaced by previously undetected species (Hart et al., 2001; Husband et al., 2002).

The present study also revealed a significant correlation between spore density and root colonization. This may be due to edaphic or climatic factors, root morphology of host plant, and germination of AM propagules (Beyene et al., 2016; Zangaro et al.,2005). Similarly, RA and IF showed significant positive correlation at both the sites indicating that AM species producing more spores usually had a wider distribution, while species with small geographic ranges usually produced fewer spores as reported earlier (Zhao and Zhao, 2007).

Table 5: Diversity measurements of AM fungal communities at the two mine sites.

\begin{tabular}{|l|c|c|}
\hline \multirow{2}{*}{\multicolumn{1}{|c|}{ Ecological parameters }} & \multicolumn{2}{c|}{ Values } \\
\cline { 2 - 3 } & Site 1 & Site 2 \\
\hline Shannon-Weiner index (H) & 2.16 & 2.07 \\
\hline Simpsons index of dominance (D) & 0.84 & 0.81 \\
\hline Evenness (E) & 0.76 & 0.73 \\
\hline
\end{tabular}

It is observed that the Shannon Wiener diversity $(\mathrm{H})$ was higher at site 1 . The distribution of AM species was uniform at both the study sites. According to Bever et al., (1996) the pattern of unevenness in spore density is due to differences in sporulation pattern of AM species.

\section{CONCLUSION}

From a restoration perspective, it is very much essential to understand the factors leading to stabilization of mine wastelands and conditions under which plants establish to become stable plant communities. In the present study, an appreciable amount of AM fungal diversity has been recorded in plants growing on stabilized iron ore mine dumps. However, further studies are required to understand seasonal variations and sporulation patterns of AM fungi at different phenology of host plants.

\section{REFERENCES}

Bever, J.D., Morton, J.B., Antonovics, J. and Schultz, P.A. 1996. Host dependent sporulation and species diversity of arbuscular mycorrhizal fungi in a mown grassland. Journal of Ecology 84 (1): 71-82.

Beyene, D., Fassil, A. and Zebene, A. 2016. Diversity and abundance of arbuscular mycorrhizal fungi under different plant and soil properties in Sidama, Southern Ethiopia. Advances in Bioscience and Bioengineering 4 (3): 16-24.

Blaszkowski, J. 2012. Glomeromycota. W. Szafer Institute of Botany, Polish Academy of Sciences, Kraków. 297pp.

Bolan, N.S. 1991. A critical review on the role of mycorrhizal fungi in the uptake of phosphorus by plants. Plant and Soil 134: 189-207.

Bray, R.H. and Kurtz, L.T. 1945. Determination of total organic carbon and available forms of phosphorus in soil. Soil Science 59: 39-45.

de Miranda, J.C.C. and Harris, P.J. 1994. The effect of soil phosphorus on the external mycelium growth of arbuscular mycorrhizal fungi during the early stages of mycorrhiza formation. Plant and Soil 166: 271280 .

Finlay, R.D. 2008. Ecological aspects of mycorrhizal symbiosis: with special emphasis on the functional diversity of interactions involving the extraradical mycelium. Journal of Experimental Botany 59: 1115-1126.

Gerdemann, J.W. and Nicolson, T.H. 1963. Spores of mycorrhizal Endogone species extracted by wet sieving and decanting. Transactions of the British Mycological Society 46: 235-244.

Ghose, M.K. 1989. Land reclamation and protection of environment from the effect of coal mining operation. Mine technology 10 (5): 35-39. 
Gianinazzi, S., Gollotte, A., Binet, M.N., van Tuinen, D., Redecker, D. and Wipf, D. 2010. Agroecology: the key role of arbuscular mycorrhizas in ecosystem services. Mycorrhiza 20: 519-530.

Giovannetti, M., Avio, L. and Sbrana, C. 2010. Fungal spore germination and pre-symbiotic mycelial growth physiological and genetic aspects. In: Arbuscular Mycorrhizas: Physiology and Function. (Eds.: Koltai, H. and Kapulnik, Y.). Springer Dordrecht Heidelberg London New York 3-32.

Hanway, J.J. and Heidel, H. 1952. Soil analysis method as used in Iowa State College soil testing laboratory. Iowa State College of Agriculture 57: 1-31.

Hart, M.M., Reader, R. J. and Klironomos, J. N. 2001. Lifehistory strategies of arbuscular mycorrhizal fungi in relation to their successional dynamics. Mycologia 93: 1186-1194.

Husband, R., Herre, E.A., Turner, S.L., Gallery, R. and Young, J.P.W. 2002. Molecular diversity of arbuscular mycorrhizal fungi and patterns of host association over time and space in a tropical forest. Molecular Ecology 11: 2669-2678.

Jasper, D.A., Robson, A.D. and Abbott, L.K. 1988. Revegetation in an iron-ore mine - nutrient requirements for plant growth and the potential role of vesicular-arbuscular (VA) mycorrhizal fungi. Australian Journal of Soil Research 26: 497-507.

Karthikeyan, A. and Krishnakumar, N. 2012. Reforestation of bauxite mine spoils with Eucalyptus tereticornis Sm. seedlings inoculated with arbuscular mycorrhizal fungi. Annals of Forest Research 55: 207-216.

Khan, A.G. 2004. Mycotrophy and its significance in wetland ecology and wetland management. In: Developments in Ecosystems volume 1. (Ed.: Wong, M.H.). Elsevier, Northhampton, UK, 97-114.

Khan, A.G. 2005. Role of soil microbes in the rhizospheres of plants growing on trace element contaminated soils in phytoremediation. Journal of Trace Elements in Medicine and Biology 18 (4): 355-364.

Kikvidze, Z., Armas, C., Fukuda, K., Martínez-García, L.B., Miyata, M., Oda- Tanaka, A., Pugnaire, F.I. and Wu, B. 2010. The role of arbuscular mycorrhizae in primary succession: differences and similarities across habitats. Web Ecology 10: 50-57.

Kullu, B. and Behera, N. 2012. Status and diversity of vesicular arbuscular mycorrhiza in different age series spongeiron solid waste dumps with respect to reclamation. The Bioscan 7: 539-542.

Kundu, N.K. and Ghose, M.K. 1997. Soil profile characteristic in Rajmahal coalfield area. Indian Journal of Soil and Water Conservation 25 (1): 2832.

Lindsay, W.L and Norvell, W.A. 1978. Development of a
DTPA soil test for zinc, iron, manganese and copper. Soil Science Society of America Journal 42: 421448.

Mummey, D.L., Stahl, P.D. and Buyer, J.S. 2002. Soil microbiological properties 20 years after surface mine reclamation: spatial analysis of reclaimed and undisturbed sites. Soil Biology and Biochemistry 34: 1717-1725.

Naithani, H.B., Sahni, K.C. and Bennet, S.S.R. 1997. Forest Flora of Goa. International Book Distributors, Dehra Dun.

Phillips, J.M. and Hayman, D.S. 1970. Improved procedures for clearing roots and staining parasitic and vesicular-arbuscular mycorrhizal fungi for rapid assessment of infections. Transactions of the British Mycological Society 55: 158-161.

Rao, R.S. 1985-86. Flora of Goa, Diu, Daman, Dadra and Nagarhaveli, 2 vols. Botanical Survey of India, Calcutta.

Read, D.J., Koucheki, H.K. and Hodgson, J. 1976. Vesiculararbuscular mycorrhiza in natural vegetation systems I; The occurrence of infection. New Phytologist 77: 641-653.

Redecker. D., Schüßler, A., Stockinger, H., Stürmer, S.L., Morton, J.B. and Walker, C. 2013. An evidencebased consensus for the classification of arbuscular mycorrhizal fungi (Glomeromycota). Mycologia 23: $515-531$

Rodrigues, B.F. and Bukhari, M.J. 1995. Occurrence of VAMF colonization in herbaceous plant species growing on iron ore mine wasteland in Goa. In: Microbial Biotechnology. (Eds.: Reddy, S.M., Srivastava, H.P., Purohit, D.K. and Reddy, S.R.). Scientific Publishers, Jodhpur, 83-86.

Rodrigues, B.F. 2000. Diversity of Arbuscular Mycorrhizal (AM) fungal species from iron ore mine wastelands of Goa. The Indian Forester 126(11): 1211-1216.

Rodrigues, B.F. and Muthukumar, T. 2009. Arbuscular mycorrhizae of Goa - a manual of identification Protocols, Goa University, Goa, India 109-135.

Sastry, M.S.R. and Johri, B.N. 1999. Arbuscular mycorrhizal fungal diversity of stressed soils of Bailadila iron ore sites in Bastar region of Madhya Pradesh. Current Science 77: 1095-1100.

Schüßler, A. and Walker, C. 2010. The Glomeromycota: A species list with new families and new genera. The Royal Botanic Garden Edinburgh, The Royal Botanic Garden Kew, Botanische Staatssammlung Munich, and Oregon State University: Create Space Independent Publishing Platform.1-58.

Shannon, C.E. and Wiener, W. 1949. The Mathematical Theory of Communication. University of Illinois Press, Urbana, USA. 
Sharma, D., Kapoor, R. and Bhatnagar, A.K. 2009. Differential growth response of Curculigo orchoides to native arbuscular mycorrhizal fungal (AMF) communities varying in number and fungal components. European Journal of Soil Biology 45 (4): 328-333.

Sheoran, A., Sheoran, V. and Poonia, P. 2008. Rehabilitation of mine degraded land by metallophytes. Mining Engineers Journal 10: 11-16.

Sheoran, V., Sheoran, A.S. and Poonia, P. 2010. Soil reclamation of abandoned mine land by revegetation: A review. International Journal of Soil, Sediment and Water 3(2): 1-20.

Simpson, E.H. 1949. Measurement of Diversity. Nature 163: 688.

Singh, A.N., Raghubanshi, A.S. and Singh, J.S. 2002. Plantations as a tool for mine spoil restoration. Current Science 82 (12): 1436-1441.

Soka, G. and Ritchie, M. 2014. Arbuscular mycorrhizal symbiosis and ecosystem processes: prospects for future research in tropical soils. Open Journal of Ecology 4:11-22.

Subbiah, B.V. and Asija, G.L. 1956. A rapid procedure for the determination of available nitrogen in soils. Current Science 25: 259-260.
Tinker, P.B. 1975. Effects of vesicular-arbuscular mycorrhizas on higher plants. Symposium of the Society for Experimental Biology 29:325-349.

Vartak, V. D. 1966. Enumeration of Plants from Gomantak, MACS, Pune.

Wang, B. and Qui, Y.L. 2006. Phylogenetic distribution and evolution of mycorrhizae in land plants. Mycorrhiza 16: 299-363.

Willis, A., Rodrigues, B.F. and Harris, P.J. 2013. The Ecology of Arbuscular Mycorrhizal Fungi. Critical Reviews in Plant Sciences 32:1-20.

Zangaro, W., Nishidate, F.R., Camargo, F.R.S., Romagnoli, G.G. and Vandresen J. 2005. Relationships among arbuscular mycorrhizas, root morphology and seedling growth of tropical native woody species in southern Brazil. Journal of Tropical Ecology 21: 529-540.

Zhao, D.D. and Zhao, Z.W. 2007. Biodiversity of arbuscular mycorrhizal fungi in the hot-dry valley of the Jinsha River, Southwest China. Applied Soil Ecology 37: 118-128. 